Onderzoeksrapport $\mathbb{N}^{\circ} 7808$

\title{
HEURISTIC PROCEDURES FOR THE SINGLE OPERATION, MULTI-ITEM LOADING PROBLEM.
}

by

M.R. LAMBRECHT ${ }^{*}$

H. VANDERVEKEN.

Wettelijk Depot : D/1978/2376/11.

* Katholieke Universiteit Leuven, Department of Applied Economics. This work was supported by the "Fonds Derde Cyclus Project : MultiPeriod Production Planning : Investigation of Dynamic Lot-Size Niodels for Complex Production systems".

To authors are greatly indebted to Prof. J. Vander Eecken and Prof. W. Herroelen for their valuable suggestions. 


\title{
HEURISTIC PROCEDURES FOR THE SINGLE OPERATION, MULTI-ITFM LOADING PROBLEA.
}

\author{
M.R. LAMBRECHT \\ H. VANDERVEKEN
}

I. INTRODUCTION

This paper considers the planning of individual machine groups or work centers producing many different products such as components, subassemblies or assemblies. This production planning problem typically arises in a Material Requirements Planning (MRP) context. A central func.... tion of any MRP system is to determine what components, sub-assemblies or assemblies are required, how many, when and where they are needed. The net requirements or planned orders for manufactured items generate load profiles showing the overloads and underloads on each work center. These load profiles must be adjusted in order to meet the available capacity which consists of available manpower and limited resources. Since the input to the work centers is erratic, the machine loads may vary considerably through time making the scheduling problem very difficult. Moreover: there is usually a high set-up cost and a substantial loss in productive time by changing production from one item to the other. The loading models presented in this paper prepare a loading plan that meets the company's production demand and minimizes production and inventory holding costs for each time period over a number of future time periods.

The scheduling problem described above is known as the multi-item, multi-period lot size problem. The problem can be stated more formally in the following way : consider a single machine shop producing many different items $i, i=1, \ldots, N$, so as to meet a known requirement schedule $\mathrm{d}_{i t}$, over a finite planning horizon $\mathrm{t}=1, \ldots \mathrm{T}$ and remain within the known 
capacity limitations $L_{r t}$ for a number of resources $r, r=1, \ldots R$. The per unit capacity absorption of product $i$, resource type $r$, is denoted by $k_{i r}$. Whenever a production run is started at the beginning of period $t$ a set-up charge $s_{i t}$ is incurred. Furthermore, there is a linear production cost $v_{i t}$ and inventory $I_{i t}$ carried from period to period $t+1$ is penalized at a rate of $h_{i t}$ per unit per period. The number of units produced in period $t$, product $i$ is denoted by $x_{i t}$. The objective is to minimize total costs subject to the demand and capacity constraints.

Mathematically we obtain :

(P) Minimize $\sum_{i=1}^{\mathbb{N}} \sum_{t=1}^{T}\left[s_{i t} \delta\left(x_{i t}\right)+v_{i t} x_{i t}+h_{i t} I_{i t}\right]$

Subject to

(1)

$$
\dot{I}_{i, t-1}+x_{i t}-I_{i t}=d_{i t} \quad \begin{aligned}
& i=1, \ldots N \\
& t=1, \ldots T
\end{aligned}
$$

(2) $\sum_{i=1}^{N} k_{i r} x_{i t} \leqslant I_{r t}$

$$
\begin{aligned}
& r=1, \ldots R \\
& t=1, \ldots \mathrm{T}
\end{aligned}
$$

$$
\begin{aligned}
& I_{i 0}=I_{i T}=0 \\
& x_{i t} \geqslant 0, I_{i t} \geqslant 0
\end{aligned}
$$

(3) $\delta\left(x_{i t}\right)=0$ if $x_{i t}=0$

$$
=1 \text { if } x_{i t}>0
$$

Constraint set (1) states that the inventory at the end of period $t-1$ plus production in period $t$ equals demand in period $t$ plus ending 
inventory period t. Constraint set (2) says that the total capacity absorption in any period $t$ must be less than the arailable capacity for alI resources. Constraints (2) may also include a term to allow for losses of productive time due to production change-over. The $(0,1)$ variables $\delta\left(x_{i t}\right)$ are defined as follows : if production is positive $\delta\left(x_{i t}\right)$ is equal to 1 and a set-up cost is incurred, otherwise the variable takes on the value $O$ and no set-up costs are included. The resulting model is of the mixed-integer type and from a computational point of view extremely difficult to solve.

In section II, the structure of the optimal solution is characterized and in section III two heuristic procedures are analyzed. The performance of both heuristics for a sample of test problems is examined in section IV. 
II. CHARACTERIZATION OF THE OPTIMAL SOLUTION.

The multi-item lot size problem has attracted much attention during the past two decades. Several linear programming models have been suggested to solve the problem. Alan Manne [12] proposed the following procedure : instead of searching for the optimal solution among all feasible production plans, he restricts his attention to those production programs satisfying the following condition :

$$
x_{i t} \cdot I_{i, t-1}=0, i=1, \ldots N, t=1, \ldots T \text {. }
$$

Condition (4) states that if production in period $t$, product $i\left(x_{i t}\right)$ is positive then $I_{i, t-1}$ must be zero and vice versa. For each product there are $2^{T-1}$ schedules satisfying (4). Without loss of generality the initial inventory ( $I_{i o}$ ) is assumed to be zero. Table 1 illustrates condition (4) for a 3-period problem.

\begin{tabular}{|cc|ccc|}
\hline & $\mathrm{d}_{\mathrm{t}}$ & 10 & 20 & 30 \\
\hline Schedule & 1 & 10 & 20 & 30 \\
& 2 & 30 & 0 & 30 \\
& 3 & 10 & 50 & 0 \\
& 4 & 60 & 0 & 0 \\
\hline
\end{tabular}

Table 1

Each of the $2^{T-1}$ schedules for product $i$ can now be denoted by a variable, for $\mathbb{N}$ products, we have a problem with $\mathbb{N} \cdot 2^{T-1}$ variables. Next an LP problem is formulated in order to select for each product one of the $2^{T-1}$ production schedules. There are two reasons why the above approach seems impracticable. Firstly, the number of variables can become extremely large and secondly, the solution to the IP problem 
may result in "mixed" production schedules that is to say schedules obtained as combinations of two or more production schedules satisfying (4). However, mixed production schedules are unrealistic because fractional set-up costs are meaningless.

The main objection from a theoretical point of view is the fact that the IP-model of Manne practically always results in approximate solutions. This loss in optimality is due to the fact that dominant schedules i.e., schedules which must be taken into consideration in order to find the optimal production plan constitute in fact a much wider class of production schedules than the ones characterized by (4). Consider the following small it period -3 product problem, with one capacity constraint $(R=1)$

\begin{tabular}{|c|c|c|c|c|}
\hline$i^{d^{i t}{ }^{t}}$ & 1 & 2 & 3 & 4 \\
\hline 1 & 20 & 30 & 40 & 10 \\
\hline 2 & 20 & 10 & 10 & 10 \\
\hline 3 & 25 & 30 & 30 & 30 \\
\hline i & 1 & 2 & 3 & \\
\hline$s_{i t}=s_{i}, \forall t$ & 70 & 90 & 200 & \\
\hline$v_{i t}=v_{i}, v t$ & 30 & 40 & 50 & \\
\hline$h_{i t}=h_{i}$, Tt & 3 & 4 & 5 & \\
\hline$k_{i}$ & 5 & 4 & 6 & \\
\hline
\end{tabular}

The optimal solution for this problem is given in the table below. 


\begin{tabular}{|c|c|c|c|c|}
\hline${ }^{x_{i t}} t^{t}$ & 1 & 2 & 3 & 4 \\
\hline 1 & 20 & 42 & 38 & 0 \\
\hline 2 & 30 & 0 & 20 & 0 \\
\hline 3 & 25 & 30 & 30 & 30 \\
\hline $\begin{array}{l}\text { Capacity } \\
\text { absorption }\end{array}$ & 370 & 390 & 450 & 180 \\
\hline Constraint & 450 & 400 & 450 & 300 \\
\hline
\end{tabular}

Tabj.e 2.

The best (but suboptimal) Wagner-Whitin type of solution is given in the next table.

\begin{tabular}{|c|rrrr|}
\hline \multicolumn{1}{|c|}{ i $^{x_{\text {it }}}$} & 1 & 2 & 3 & 4 \\
\hline 1 & 20 & 30 & 50 & 0 \\
2 & 40 & 0 & 0 & 10 \\
3 & 25 & 30 & 30 & 30 \\
\hline Capacity & 410 & 330 & 430 & 220 \\
\hline absorption & & & & \\
\hline Constraint & 450 & 400 & 450 & 300 \\
\hline
\end{tabular}

Table 3.

To shed some light on these issues we characterize in what follows the extreme points of the solution set (P).

The constraints of problem (P) can easily be represented by means of a network. This network representation is given in figure 1 : 


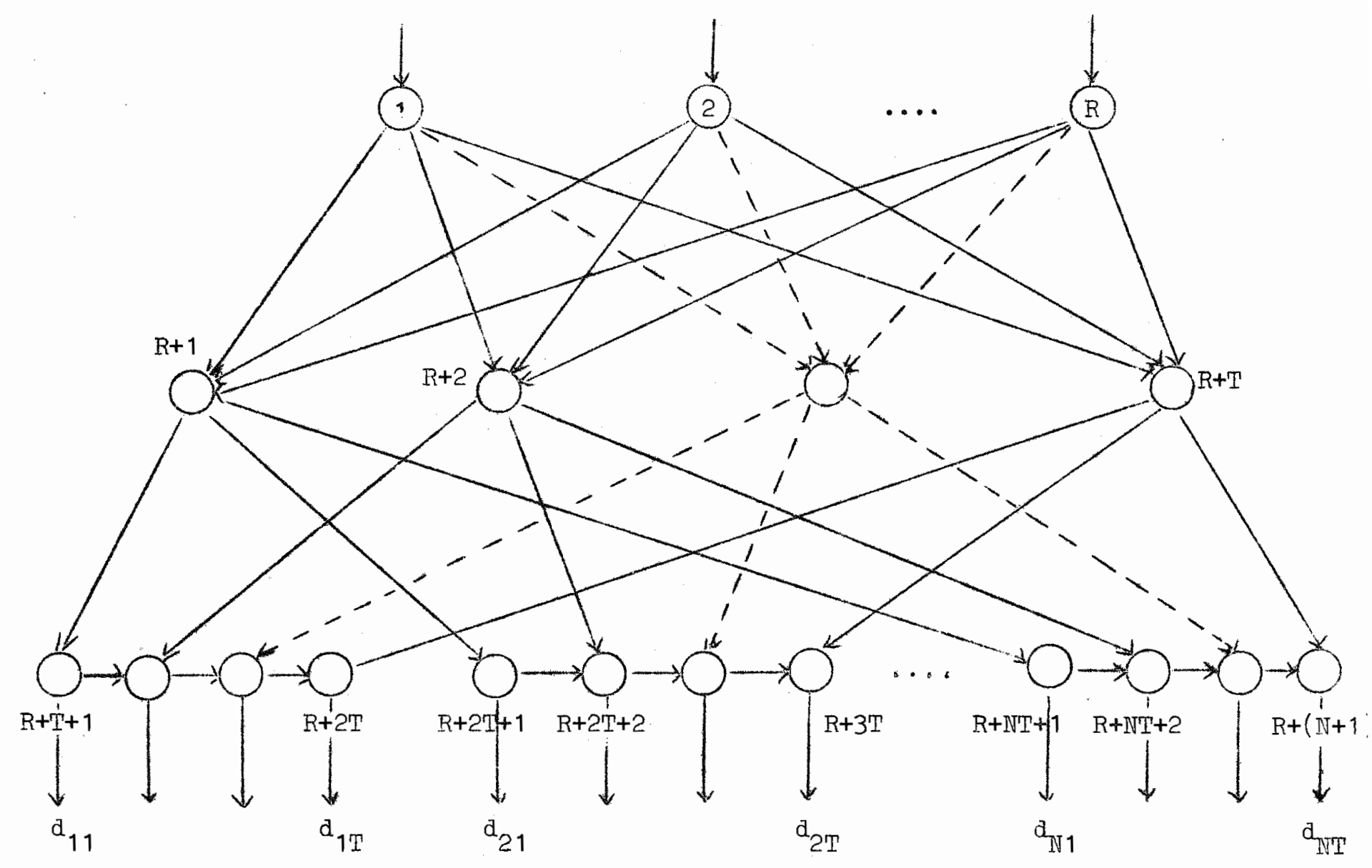

Figure 1.

The input into nodes $1, \ldots, R$ equals $\sum_{i=1}^{N} \sum_{t=1}^{T} k_{i r} d_{i t}, r=1, \ldots R$. The flow over the arcs connecting nodes $(1, \ldots, R)$ and $(R+1, \ldots, R+T)$ represents the aggregate flow in each time period, for each resource type and equals $\sum_{i=1}^{\mathbb{N}} k_{i r} x_{i t}, t=1, \ldots T, r=1, \ldots R$. This flow is restricted to be at most $L_{r t}$ units (constraint set (2)).

The flow over the arcs connecting nodes $(R+1, \ldots, R+T)$ and $(R+T+1, \ldots$, $R+(N+1) T$ ) represents $x_{i t}$ the amount produced in each time period for each 
product. Constraint set (1) is represented in the network by means of the in and outflows into nodes $(R+T+1, \ldots, R+(N+1) T)$.

We clearly have two types of flows in the network above, first, the aggregate flow between the nodes $(1, \ldots, R)$ and $(R+1, \ldots R+T)$ and second, the individual product flow between nodes $(R+1, \ldots R+T)$ and $(R+T+1, \ldots$, $\mathrm{R}+(\mathrm{N}+1) \mathrm{T}$ ). The aggregate flow is capacitated whereas the individual product flow is not (except for the conditions $x_{i t} \geqslant 0, I_{\text {it }} \geqslant 0$ ).

The constraint set of $(P)$ is bounded and the objective function is concave so that its minimum will be achieved at one of the extreme points. In a networls terminology, we shall refer as in [17] to such extreme points as extremal flows. Roughly speaking, a flow in an uncapacitated network is extremal if it is constructed in a way that prevents the formation of positive loops. A loop is defined as a sequence of nodes and arcs obtained by selecting one of the arc orientations and for which the first and last node coincide. A loop is positive if all of its arcs carry positive flows. It is also well known [4] that a feasible flow in a capacitated network is extremal if and only if each of its positive loops contains at least one saturated arc, i.e. an arc whose flow is at capacity.

In the network of figure 1, loops can be formed in two ways :

(1) between the aggregate and invidual product level, e.g. the loop formed by the sequence of nodes $(1, R+1, R+T+1, R+T+2, R+2,1)$ an by the ares $(1, R+1),(R+1, R+2 T+1),(R+2 T+1, R+2 T+2),(R+2, R+2 T+2)$ and $(1, R+2)$

(2) on the individual product level, e.g. the loop formed by the sequence of nodes $(R+1, R+T+1, R+T+2, R+2, R+N T+2, R+N T+1, R+1)$ and by the arcs $(R+1, R+T+1),(R+T+1, R+T+2),(R+2, R+T+2),(R+1, R+N T+1),(R+N T$, $R+N T+2)$ and $(R+2, R+N T+2)$.

Production programs satisfying the Wagner-Whitin condition $x_{i t} \cdot I_{i, t-1}=0$, will always be extreme flows. Such production programs always prevent the formation of positive loops. 
If on the other hand $x_{i t} \cdot I_{i, t-1} \neq 0$ then two conzitions must be satisfied in order to have an extreme $f I J$. We call these conditions respectively the aggregate flow condition and the individual product flow condition.

In order to clarify these two conditions some additional terminology is needed.

(a) $\mathrm{N}^{\circ}$ is defined as the set of products for which $x_{i t} \cdot I_{i, t-1}=0$, 1 t and $\mathrm{N}^{*}$ is defined as the set of products for which this property is not satisfied $\left(\mathbb{N}^{*} \mathrm{UH}^{\circ}=\mathrm{N}\right)$.

(b) $e_{r t}$ is defined as the excess or slack capacity in period $t$, resource type $r$, i.e.,

$$
e_{r t}=I_{r t}-\sum_{i=1}^{N} k_{i r} \cdot x_{i t}, \quad \begin{aligned}
t & =1, \ldots T \\
r & =1, \ldots R .
\end{aligned}
$$

(c) A regeneration point, $k$, for product $i$ is defined as a period with zero ending inventory or $I_{i k}=0$.

(d) Between each pair of successive regeneration points $(k, l)$ for product $i, 0 \leqslant k<I \leqslant T$, we have that $I_{i k}=I_{i l}=0$ and $I_{i p}>0, p=k-1, \ldots$, 1-1. Production between successive regeneration points $(k, 1)$ may either be zero or strictly positive. Assume that production is zero in $q$ periods with $0 \leqslant q \leqslant(1-k)$ and positive in the remaining $(1-k-q)$ periods.

Consider a product iEN $\mathrm{N}^{\bar{*}}$.

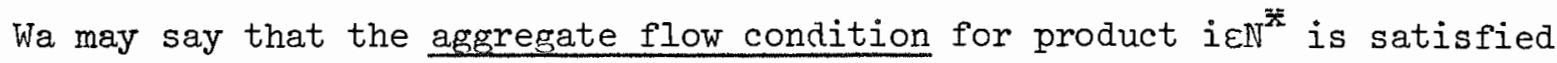
if for each pair of regeneration points $(k, l)$, with $q$ periods of zero production and $(1-k-q)$ periods of positive production, we have that for at least one resource type $r$, there is at most one of the $(1-k-q)$ periods with positive excess capacity. 
The aggregate flow condition is very similar to the property developed by Florian and Klein [5], [9], [10] for the siugle item capacity constrained dynamic lot size problem. Since we are dealing with a network with $R$ sources (resource types), the above condition must hold for at least one resource type $[17]$.

In the above example $\mathbb{N}^{*}=\{1\}$. For product 1 however, the aggregate flow condition is satisfied, since for the pair of regeneration points $(1,4)$, with zero production in period $4(q=1)$ and positive production in periods 2 and $3(1-k-q=2)$, there is only one period (namely period 2) with positive excess capacity.

If there is more than one product in the set $\mathrm{N}^{*}$, then the aggregate flow condition is not enough to prevent the formation of positive loops. A second condition, the individual product flow condition, must be satisfied.

Consider products $i^{\prime}, i^{\prime \prime}$ and $i^{\circ}$ belonging to $\mathbb{N}^{*}$. The production and ventory flow ketween the pairs of regeneration points for products belonging to $\mathrm{N}^{*}$ may form positive loops.

The detection of positive loops can be illustrated by means of the following graphical aid (see figure 3 ).

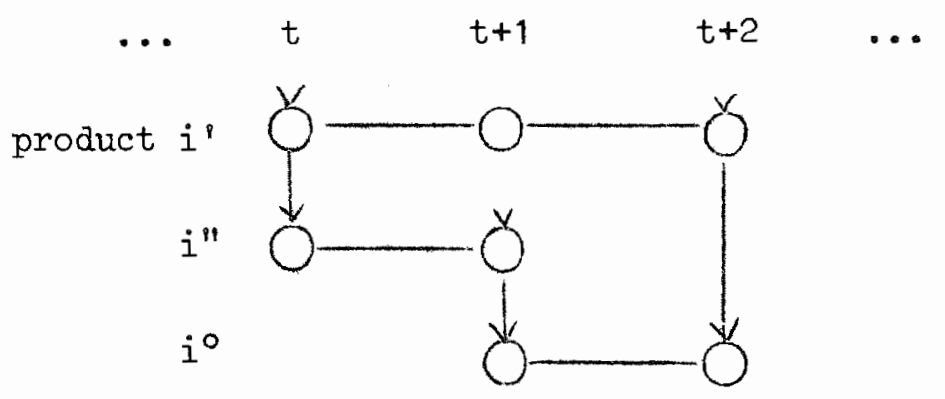

figure 2 
Consider the following pairs of regeneration points $(t-1, t+2)$, $(t-1, t+1)$ and $(t, t+2)$ for respectively products $i^{p}, i^{\prime \prime}$ and $i^{\circ}$. The horizontal line segments represent positive inventory between the pairs of regeneration points. Hodes labeled ( $\zeta$ ) refer to periods with positive production. Vertical lines are drawn only between labeled nodes.

If through this procedure a connected path is formed, then a positive loop will be formed between the regeneration points of the products belonging to $\mathrm{N}^{* *}$. Note that this procedure enables us to detect all positive 1oops.

We say that the individual product flow condition is satisfied if no such connected path can be formed.

It is obvious that this condition has a great influence on the construction of solution algorithms.

By construction, $D^{*}$, the set of feasible production plans $X^{*}$ :

$$
x^{*}=\left\{\left(x_{i t}, I_{i t}\right), \forall i, t \mid x_{i t} \cdot I_{i, t-1}=0, \frac{I t}{v}, i \in N^{\circ} \text { or } x_{i t} \cdot I_{i, t-1} \neq 0\right.
$$

for $i \varepsilon N^{*}$ and for which the aggregate and individual product flow conditions are satisfied?,

corresponds to the finite set of extreme points. 
III. HEURISTIC APPROACHES.

The objective of heuristic approaches is to find "good" solutions for complex decision problems with less computational effort compared to the optimal seeking algorithms. Several heuristics have been proposed [2] [6], [8], [11], [13] for solving the multi-item scheduling problem. All those heuristics are based on Manne's LP formulation but since the number of variables usually turns out to be very large for practical problems, these methods have not been very successful. Moreover, these heuristics, require input (requirements, capacities, etc...) over the complete planning horizon which is not desirable in an environment subject to many changes. Eisenhut [3] on the other hand proposed a heuristic based on marginal analysis and at the same time lot sizes are determined on a period by period basis. His approach is basically myopic in the sense that only limited information is needed to make the current decision. Such sequential approaches have also disadvantages especially for problems with capacity considerations. The main drawback of the Eisenhut heuristic lies in the fact that it can end up with an infeasible solution. We therefore propose to extend his heuristic by incorporating a feedback mechanism to escape from infeasibility.

\section{A. The Eisenhut Heuristic.}

The Eisenhut approach starts from a requirements matrix whose elements $a_{i t}, i=1, \ldots N, t=1, \ldots T$ are the net requirements for the different products over the planning horizon, and then tries to group requirements of different periods in the same lot without exceeding the capacity constraints. For each requirement, $d_{i t}$, a coefficient is calculated indicating if cost reductions are possible by including requirements of later periods in the 
current production order. The derivation of this "appreciation factor" goes as follows:

$$
C_{i}(t)=\frac{s_{i}+I_{i}(t)}{t}, \quad i=1, \ldots N \text {, where }
$$

$c_{i}(t)$ is the combined set-up and inventory holding cost per period for product $i$, for a production order including production requirements for periods 1 up to $t$, with $t \leqslant T$

$s_{i}$ denotes the set-up cost for product $i$

$I_{i}(t)$ stands for the cumulative inventory holding cost corresponding to an order including requirements for periods 1 up to $t$, or

(8) $\quad I_{i}(t)=h_{i} \sum_{k=1}^{t}(k-1) d_{i k} \quad$ with

$h_{i}$ as the inventory holding cost per unit, per period for product $i$.

The objective is to minimize $C_{i}(t)$. We therefore calculate the first derivative of ( 7 ).

(9) $\quad C_{i}(t)=\frac{I_{i}(t)}{i}-\frac{I_{i}(t)}{t^{2}}-\frac{s_{i}}{t^{2}}$

where $I !(t)$ is the change in inventory holding cost due to incorporating the requirement $d_{i t}$ in the present lot, or

(10) $\quad I_{i}^{\prime}(t)=h_{i}(t-1) d_{i t}$

Since $\sum_{k=1}^{t}(k-1)=\frac{(t-1) t}{2}$ or $(t-1)=\frac{2}{t}\left[\sum_{k=1}^{t}(k-1)\right]$ 
We can rewrite expression (10) as follows:

(11) $\quad I_{i}^{\prime}(t)=\frac{2 h_{i}}{t}\left[\sum_{k=1}^{t}(k-1) d_{i t}\right]$

Assuming that demand is constant through time, expression (11) reduces to

(12) $\quad I_{i}^{\prime}(t)=\frac{2}{t} I_{i}(t)$

Substituting (12) in (9), we obtain

$$
C_{i}(t)=\frac{2}{t^{2}} I_{i}(t)-\frac{I_{i}(t)}{t^{2}}-\frac{s_{i}}{t^{2}}
$$

or

(13) $\quad C_{i}(t)=\frac{I_{i}(t)-s_{i}}{t^{2}}$

Let $C_{i}^{!}(t)$ approach zero, and since $t$ has discrete values we find $t^{*}$, the best unconstrained order cycle if

(14) $\quad I_{i}\left(t^{*}\right) \leqslant s_{i}$ and $I_{i}\left(t^{*}+1\right)>s_{i}$

Note that criterium (14) is known as the Part Period Balancing criterium.

Instead of using (13) Eisenhut prefers the following coefficient

(15) $\quad U_{i}(t)=-\frac{C_{i}^{p}(t)}{d_{i t}}=\frac{s_{i}-I_{i}(t)}{t^{2} \cdot d_{i t}} \quad i=1, \ldots N$

such that for $t<t^{*}$ the term $U_{i}(t)$ will be positive, indicating a cost reduction and for $t>t^{*}, U_{i}(t)$ will be negative indicating a cost increase. 
Note that in $(15)$ the coefficient $C_{i}(t)$ is devided by $a_{i t}$ so that the appreciation factor is now expressed in costs per unit per period (our computational experience indicate that the devision by $d_{i t}$ is of no importance.)

The procedure can be sumarized as follows :

Step 1 : Construct the requirement matrix, the rows $i, i=1, \ldots \mathbb{N}$ stand for the different products and the columns $t, t=1, \ldots$. for the different time periods, the elements $(i, t)$ indicate requirements $d_{i t}$ for product $i$, period $t$.

Step_2 : Compute for each product $i$ and each period $t$ (except the first period, who's requirements must be satisfied), the coefficient $U_{i}(t)$, omitting negative values. If in the current period the net requirements for product $i$ are zero, i.e. $d_{i t}=0$, then product $i$ is disregarded since it will not be advantageous to start a production run for that item. Postponing such a run will result in lower inventory carrying charges.

Step 3 : In order to determine the lot sizes for period $t$ (starting at $t=1$ ) the following steps must be carried out. For each product $i$, the requirements of period $t$ must be satisfied and hence included in the lot. Next, for each product, scan the appreciation factors of the most recent requirements which have not yet been included in the lot of period $t$. Select the highest positive $U_{i}(t)$ and add the corresponding requirements to the lot of period $t$ except if insufficient capacity is available. If capaicity should be exceeded, the corresponding product is discarded and the next highest positive coefficient is selected. This step is repeated until capacity is reached for one of the resources, or until no positive coefficients remain. Note that each time that the order size is increased, the slack capacity $e_{r t}$ must be updated. 
Step 4 : Update the requirements matrix by deleting the requirements of period $t$ and all requirements included in the production or lers of period $t$.

Step_5 : Repeat steps 2 to 4 until the requirements matrix is reduced to one column, namely the requirements of period $T$

The above algorithm may best be illustrated by means of the problem example given in Section II.

Step 1

\begin{tabular}{|c|c|c|c|c|}
\hline$i$ & 1 & 2 & 3 & 4 \\
\hline 1 & 35 & 40 & 30 & 37 \\
\hline 2 & 24 & 27 & 29 & 22 \\
\hline 3 & 0 & 50 & 47 & 42 \\
\hline
\end{tabular}

Step 2

\begin{tabular}{c|cccc}
$i$ & 1 & 2 & 3 & 4 \\
\hline 1 & $35^{*}$ & $40^{0,375} 30^{0}$ & $37^{-}$ \\
2 & $24^{*}$ & $27^{0,611} 29^{-}$ & $22^{-}$ \\
3 & $0^{*}$ & $50^{*}$ & $47^{*}$ & $42^{*}$
\end{tabular}

- The coefficient for the requirements $d_{i 1}, i=1,2,3$ need not be determined because they must be included in the production orders of period 1 . The remaining capacities are :

$$
\begin{aligned}
e_{11} & =230-(35 \times 2+24 \times 3)=88 \\
e_{21} & =170-(35 \times 1+24 \times 2)=87 \\
\cdot U_{1}(2) & =\frac{100-40}{2^{2} \cdot 40}=0,375, U_{2}(2)=\frac{120-27 \times 2}{2^{2} \cdot 27}=0,611
\end{aligned}
$$


$\mathrm{U}_{3}(2), \mathrm{U}_{3}(3), \mathrm{U}_{3}(4)$ are not computed because demand in period 1 is zero. $\mathrm{U}_{1}(3)=\frac{100-40-60}{3^{2} \times 30}=0$

All other coefficients are negative.

Step $3: U_{2}(2)$ is the highest positive coefficient, the corresponding requirements are 27 . We increase the lot of product 2 with 27 units and reduce the available capacities correspondingly.

$$
\begin{aligned}
& e_{11}=88-(27 \times 3)=7 \\
& e_{21}=87-(27 \times 2)=33
\end{aligned}
$$

The next highest coefficient is $U_{1}(2)$, but the production order for product 1 cannot be increased by 40 units as there is not enough capacity available.

Step 4,2

\begin{tabular}{l|rll}
$i$ & 2 & 3 & 4 \\
\hline 1 & $40^{*}$ & $30^{0,583}$ & $37^{-}$ \\
2 & $0^{*}$ & $29^{*}$ & $22^{*}$ \\
3 & $50^{*}$ & $47^{0,580}$ & $42^{-}$
\end{tabular}

$$
\begin{aligned}
& e_{12}=200-(40 \times 2+50 \times 1)=70 \\
& e_{22}=180-(40 \times 1+50 \times 1)=90
\end{aligned}
$$

Step 3 : Highest coefficient : $\mathrm{U}_{1}(3)$, increase the lot size for product 1 , period 2 by 30 units; the remaining capacities are

$$
\begin{aligned}
& e_{12}=70-(30 \times 2)=10 \\
& e_{22}=90-(30 \times 1)=60
\end{aligned}
$$


The second highest coefficient is $U_{3}(3)$, the order size cannot be increased by 47 units since capacity limits will be exceeded for resource 1 .

Step 4,2

\begin{tabular}{l|ll}
$i$ & 3 & 4 \\
\hline 1 & $0^{* x}$ & $37^{z x}$ \\
2 & $29^{x}$ & $22^{0,86}$ \\
3 & $47^{* x}$ & $42^{0,74}$
\end{tabular}

$$
\begin{aligned}
& e_{13}=270-(29 \times 3+47 \times 1)=136 \\
& e_{23}=160-(29 \times 2+47 \times 1)=55
\end{aligned}
$$

Step 3 : Highest coefficient : $U_{2}(4)$, increase lot size for product 2 , period 3 with 22 units

$$
\begin{aligned}
& e_{13}=136-(22 \times 3)=70 \\
& e_{23}=55-(22 \times 2)=11
\end{aligned}
$$

The net requirements corresponding to $\mathrm{U}_{3}(4)$, the next highest coefficient, cannot be scheduled because of insufficient capacity.

Step 4,5

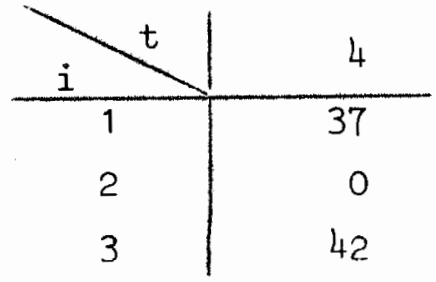

The resulting production plan is as follows: 


\begin{tabular}{|c|c|c|c|c|c|}
\hline & i & 1 & 2 & 3 & 4 \\
\hline & 1 & 35 & 70 & 0 & 37 \\
\hline & 2 & 51 & 0 & 51 & 0 \\
\hline & 3 & 0 & 50 & 47 & 42 \\
\hline \multirow[t]{2}{*}{$\begin{array}{c}\text { Capacity } \\
\text { used }\end{array}$} & $r=1$ & 223 & 190 & 200 & 116 \\
\hline & $r=2$ & 137 & 120 & 149 & 79 \\
\hline
\end{tabular}

The alert reader should realize that :

a) The Eisenhut heuristic can easily handle problems where set-up times. are important, simply by subtracting the set-up times from the available capacity each time that a production run is started.

b) The production programs found by the Eisenhut heuristic always satisfy the condition

$$
x_{i t} \cdot I_{i, t-1}=0
$$

That means that the heuristic does not fully exploit the characteristics of optimal solutions as explained in section II.

B. Extended Eisenhut Heuristic.

\section{Feedback Mechanism.}

The Eisenhut heuristic proceeds uni-directional through the requirements matrix without feed-back capability. As a result, the heuristic can end up with an infeasible solution and this often occurs when the capacity constraints are tight and level from period to period whereas demand has somehow an erratic behavior. To cure this deficiency and thereby enhance the applicability of the Eisenhut heuristic, the following feed-back mechanism is proposed. 
If an infeasibility occurs in period $t$, i.e. if resource requirements exceed the available capacity in period $t$, then we transfer demand of that period to period $t-1$ until either one of two conditions occurs : (1) the capacity limit of one of the resource constraints is attained in period $t-1 ;(2)$ the infeasibility disappears in period $t$. If the infeasibility still remains after this phase is completed further transfers of the same or different products must be made to earlier periods in a similar fashion.

The resulting production programs practically always adhere to the characteristics of dominant schedules (section II). The aggregate flow condition is always satisfied, but the individual product flow condition may be violated for some extreme cases.

Moreover, demand is shifted backward in such a way that no new set-ups are incurred and inventory holding costs are minimal.

Let $I_{\text {INF }}$ be the amount by which the demand requirements for resource $r$, product $i$, exceed capacity in period $t$. The minimum number of units of product $i$ to be transferred in order to restore feasibility for resource $r$ is given by :

$$
A_{i r}=\left\lceil\frac{I N F_{r t}}{k_{i r}}\right\rceil
$$

Where $\lceil a\rceil$ refers to the smallest integer greater than or equal to a. For practical reasons we always transfer an integer number of units, although from a theoretical point of view optimal solutions may be noninteger.

Since feasibility must be restored for all resource constraints that are violated, we determine

$$
B_{i}=\max _{r \in R^{x i x}}\left\{A_{i r}\right\}
$$


where $R^{\mathrm{E}}$ is the set of resources for which the capacity limit is exceeded. It is clear that no more than $u_{\text {it }}$ units will be uransferred, hence, the number of units that can be transferred for a specific product is given by

$$
C_{i}=\min \left\{B_{i}, d_{i t}\right\} \text {, with } C_{i}>0 \text {, otherwise product } i \text { is discarded. }
$$

If $C_{i}$ units are transferred to period $t-1$, the inventory holding cost is increase $\hat{a}$ oy $C_{i} \cdot h_{i}$. To mirimize the costs caused by transferring units froin $t$ to period $t-1$ we select product $q$ such that :

a) there is a set-up in period $t-1$ (otherwise new set-ups would be created)

b) the increase in inventory holding cost is minimal.

In other words, $q$ is defined by

$$
\begin{aligned}
& \min _{\text {in }} C_{i} \circ h_{i}, \text { where } \mathbb{N}^{3}=\text { set of products for which there is a set-- } \\
& \text { up in period } t-1 .
\end{aligned}
$$

It is clear that the feed wback procedure may not cause an infeasibility in period $t-1$.

Let

$$
D=\min _{\frac{T}{r} r}\left|\frac{e_{r, t-1}}{k_{c r}}\right|
$$

where La」 reiers to the greatest integer less than or equal to a.

$D$ denotes the maximum number of units of product $q$ that can be transferred to period $t-1$ without violating the resource constraints for that period.

The actual number of units that will be transferred is then given by $E$, 
where

$$
E=\min \left(D, C_{q}\right)
$$

We now can end up in one of the following cases:

a) Period $t$ becomes feasible, and the Eisenhut heuristic continues with period $t+1$.

b) Period $t$ is still infeasible, and

- either $E=D$, which means that it is impossible to transfer more units of product $q$ to period $t-1$ without exceeding one of the $R$ constraints of period $t-1$. We therefore must transfer units to period t-2 using the procedure given above. Note that the infeasibility in period $t$ is reduced by $D_{x k}$ capacity units.

- or $\mathrm{E}=\mathrm{C}_{\mathrm{q}}=\mathrm{d}_{\mathrm{qt}}$ which means that in addition to the demand for prodicis i, units of other products must be transferred to period t-1. The abovementioned procedure must be repeated after disregarding product $q$.

A detailed flowchart is given in Appendix I.

As we emphasized before, the interesting features of this proce... dure are twofold : (1) solutions practically always satisfy the characte... ristics of dominant schedules ${ }^{*}$, (2) if the data of the problem allow for a feasible solution the feedback mechanism will always find one.

* The individual product flow condition is not always satisfied if backtracking is done for more than one product and for more than two periods back in time (this can happen if the capacity constraints are very tight). Minor deviations are also possible because only integral number of units are transferred. 
An example is used to illustrate the feedback procedure. Consider the following 4 product, 4 period problem with one capacity constraint.

\begin{tabular}{c|cccc} 
d $_{i t} t$ & 1 & 2 & 3 & 4 \\
$i$ & & & & \\
1 & 40 & 35 & 32 & 30 \\
2 & 30 & 35 & 37 & 34 \\
3 & 55 & 60 & 55 & 50 \\
4 & 0 & 30 & 29 & 24 \\
Capacity & & & & \\
Constraint & & & &
\end{tabular}

\begin{tabular}{l|rrr}
$i$ & $h_{i}$ & $s_{i}$ & $k_{i}$ \\
\hline 1 & 3 & 100 & 2 \\
2 & 1 & 100 & 2 \\
3 & 2 & 150 & 1 \\
4 & 1 & 50 & 3
\end{tabular}

Applying the Eisenhut heuristic for the first 2 periods results in

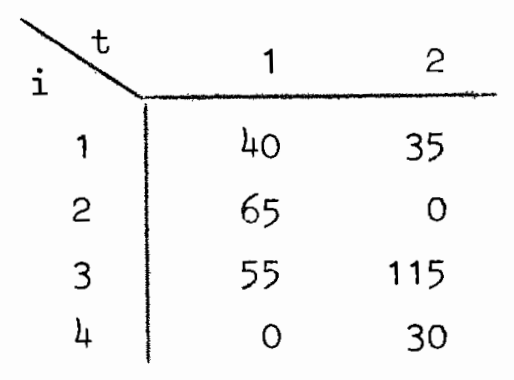

The third period however is infeasible since the capacity absorption is $32 \times 2+37 \times 2+29 \times 3=225$ and the capacity limit is 220 
Units must be transferred from period 3 to period 2. As there is only one capacity constraint, index $r$ is omitted.

$$
\begin{aligned}
& \mathrm{INF}_{3}=225-220=5, \mathrm{~N}^{*}=\{1,3,4\}, \text { for product } 2 \text { there is no set- } \\
& \mathrm{A}_{1}=\left\lceil\frac{5}{2}\right\rceil=3 \\
& \mathrm{~A}_{3}=1\left\lceil\frac{5}{1}\right\rceil 1=5 \\
& \mathrm{~A}_{4}=1\left\lceil\frac{5}{3}\right\rceil 1=2
\end{aligned}
$$

Since there is only one constraint we have that $A_{i}=B_{i}$

$$
\begin{aligned}
\mathrm{C}_{i}=\min \left\{\mathrm{B}_{i}, \mathrm{~d}_{i t}\right\} \quad \mathrm{C}_{1}=\min \{3,32\}=3 \\
\mathrm{C}_{3}=\min \{5,0\}=0, \text { excluded because no } \\
\\
\mathrm{C}_{4}=\min \{2,29\}=2
\end{aligned}
$$

$$
\begin{aligned}
& \min _{i \in \mathbb{N}^{*}} C_{i} h_{i}=\min \{3 \times 3 ; 2 \times 1\} \quad \text { selected product } q=4 \\
& D=\left\lfloor\frac{e_{2}}{k_{4}}\right\rfloor=\left|\frac{15}{3}\right|=5 ; \quad e_{2}=290-(35 \times 2+115 \times 1+30 \times 3)=15 ; \\
& E=\min \left(D, C_{4}\right)=\min (5,2)=2 .
\end{aligned}
$$

The result of the preceeding steps is that 2 units of product 4 are transferred to period 2. After this step feasibility is restored and the Eisenhut heuristic continues with period 4.

The resulting production program is 


\begin{tabular}{|c|c|c|c|c|}
\hline$x_{\text {it }} \quad t$ & 1 & 2 & 3 & 4 \\
\hline 1 & 40 & 35 & 32 & 30 \\
\hline 2 & 65 & 0 & 37 & 34 \\
\hline 3 & 55 & 115 & 0 & 50 \\
\hline 4 & 0 & 32 & 27 & 24 \\
\hline
\end{tabular}

\section{A New Appreciation Factor.}

The Eisenhut appreciation factor is derived under the assumption that requirements are constant through time. This assumption was only necessary to replace the term $I_{i}^{\prime}(t)$ by $\frac{2}{t} I_{i}(t)$ (see section III) whereas the exact derivative assuming fluctuating demand is $h_{i}(t-1) \mathrm{d}_{i t}$.

Substituting this exact term in expression (9) results in :

$$
C_{i}^{\prime}(t)=\frac{h_{i}(t-1) a_{i t}}{t}-\frac{I_{i}(t)}{t^{2}}-\frac{s_{i}}{t^{2}}
$$

Substituting for $I_{i}(t)=I_{i}(t-1)+h_{i}(t-1) d_{i t}$

We obtain

$$
C_{i}(t)=\frac{1}{t^{2}}\left[h_{i}(t-1)^{2} d_{i t}-I_{i}(t-1)-s_{i}\right]
$$

Let $C !(t) \rightarrow 0$, and since $t$ has discrete values we find the unconstrained order cycle $t^{3 \mathrm{x}}$ if

$$
h_{i}\left(t^{*}-1\right)^{2} d_{i t} \leqslant I_{i}\left(t^{*}-1\right)+s_{i}
$$


and

$$
h_{i}\left(t^{*}\right)^{2} d_{i, t^{*}+1}>I_{i}\left(t^{*}\right)+s_{i}
$$

We now prove that condition (16) is equivalent to the Silver-Meal criterium [14].

Silver-Meal [ 14 ] suggest to evaluate $C_{i}(t)$ successively for increasing values of $t$ until the following inequality is satisfied :

(17) $\quad c_{i}(t+1)>c_{i}(t)$

In [14] it is shown that (17) is equivalent to

(18) $\quad t^{2} d_{i, t+1}>\frac{s_{i}}{h_{i}}+\sum_{k=1}^{t}(k-1) d_{i k}$

After multiplying both sides by $h_{i}$ and substituting for

$$
h_{i} \sum_{k=1}^{t}(k-1) d_{i k}=I_{i t}
$$

we obtain

$$
h_{i} t^{2} D_{i, t+1}>I_{i}(t)+s_{i}
$$

thereby establishing the equivalence of the condition (16) and the Silver and Meal heuristic.

In the multi-item capacitated lot size problem we propose the following appreciation factor

(19) $U_{i}(t)=-\frac{C_{i}^{q}(t)}{d_{i t}}=\frac{I_{i}(t-1)+s_{i}-h_{i}(t-1)^{2} d_{i t}}{d_{i t} \cdot t^{2}}$ 